\title{
Comparison of Spectral Characteristics among Some Adjustable Windows
}

\author{
Weihua Dai ${ }^{*}$, Chunjie Qiao, Yueke Wang, Zhigang Huang \\ and Chao Zhou \\ College of Mechatronics Engineering and Automation, National University of Defense Technology, \\ Changsha, 410073, China
}

Keywords: Adjustable windows, Spectral characteristic, Numerical computation .

Abstract. Kinds of adjustable windows are flexibly applied in digital filter design and spectral analysis. But general description of adjustable windows for any window length are always deficient in time domain and frequency domian. Moreover, there lacks quantitative comparison of spectral characteristics among adjustable windows. In this paper, analytic expressions that suit for any window length were utilized to describe adjustable windows, including the Kaiser, Cosh, Dolph-Chebyshev, Saramäki and other ultraspherical windows. The spectral characteristics of adjustable windows were obtained through numerical computation, and their curves were numerically fitted with simple functions about adjustable parameters. The relative deviations between the computed curves and the fitted ones are normally less than 7 percent. Finally, detail comparison of spectral characteristics among adjustable windows were performed from multi-sides. It shows that the Saramäki window has better overall performance than other adjustable windows by

comparing spectral characteristics.

\section{Introduction}

Digital windows are widely used for signal spectral analysis and digital filter design. T.Saramäki has categorized windows as fixed or adjustable [1]. Window length is the only alterable parameter for fixed windows, which consist of Rectangula, Triangular, Hamming, Hanning and Blackman window and so on. The Dolph-Chebyshev [2], Kaiser [3], Saramäki [4], ultraspherical [5] and Cosh window[6] all belong to adjustable windows which have other flexible parameters besides window length.

The Dolph-Chebyshev window was first proposed by C.L.Dolph[2] to solve the problem of designing a radio antenna having optimal directional characteristics[7], it is constructed by using the well-known Chebyshev polynomials. P.Lynch[8] designed a simple optimal filter based on the Dolph-Chebyshev window to modify the initial data for numerical weather prediction models. The Dolph-Chebyshev window has explicit analytic expression[9] and equal side-lobes, but this analytic expression isn't suitable for even window length. Therefore the corresponding expressions[8] of main-lobe width or ripple ratio may also aren't suitable for even window length.

The analog Kaiser window was proposed by Kaiser[3], and the zeroth-order modified Bessel function of the first kind was suggested to approximate the prolate function[10] whose coefficients are difficult to compute. The resulting window, namely Kaiser window, then closely approximates the prolate spheroidal wave function that provides the greatest concentration of energy at low frequency. Subsequently, the digital Kaiser window was applied in nonrecursive digital filter design[11] and digital spectrum analysis[12]. For digital spectrumanalysis, the digital Kaiser window can be simply obtained from sampling the analog Kaiser window[12], but the discrete Kaiser window isn't suitable for even window length, either. A more general expression of digital Kaiser window that suit both odd and even window length was described by Oppenheim[13], but the analytic expression for calculating the spectral characteristic parameters weren't given. Moreover, power series expansions[6] are required in calculating the modified zeroth-order Bessel function which is presented in the expression.of Kaiser window. 
The Saramäki window was introduced by T.Saramäki for designing FIR filters[6]. It provides a better approximation to the discrete prolate functions than Kaiser window, and it also possesses analytic expressions while no power series expansions are required in calculating its coefficients.

The unispherical window based on the orthogonal polynomials known as the Gegenbauer or ultraspherical Polynomials[14] was first discussed by A.G.Deczky[5]. The unispherical window which is also called ultraspherical window has two other parameters besides window length, and other window functions can be approximated by this class window, but the given expression[5] only suits odd window length and it is difficult to compute the window coefficients. An effective method[15] for generating the ultraspherical window was proposed by S.W.A.Bergen and Antoniou, who later proposed a method[16] for designning the ultraspherical window that achieves prescribed spectral characteristics and an efficient method[17] for designing nonrecursive digital filters by using the ultraspherical window. They also illuminated that the Dolph-Chebyshev and Saramaki windows are both particular cases of the ultraspherical window. The coefficients expressions of right-side ultraspherical window[16] can be applied to both Dolph-Chebyshev and Saramaki window with any length, but some iterative algorithm are required for computing these expressions.

The Cosh window based on the cosine hyperbolic function was proposed by AvciK and Nacaroglu[6] who derived this class window by referring to the Kaiser window[3], as a result, they are similar in time domain representation except that the cosine hyperbolic function is applied instead of the zeroth-order modified Bessel function. The Cosh window has computational cost advantage compared with the Kaiser window due to no power series expansion in computing window coefficients, and it was used to design FIR filters by Harish Kumar, et. al. later[18]. However, the time domain representation of the Cosh window isn't suitable for even window length and there are less analytic expressions for calculating the spectral characteristics.

Besides, though performance comparison between different windows for FIR filter design and spectral analysis are familiar in many literatures[19-21], comparative study on the performance of spectral characteristics particularly among adjustable windows are always scattered and qualitative, such as, the Saramäki window provides a better approximation to the discrete prolate functions when compared with Kaiser window[4]; The ultraspherical window can achieve different side-lobe patterns while others can not[16]; The Kaiser window has better side-lobe roll-off than the Saramäki and Dolph-Chebyshev windows[6]; The Cosh window provides better side-lobe roll-off ratio characteristic for the same window length and normalized width when compared with Kaiser window[6]. However, clearer and quantitative comparison is lacking. To provide more details to the researchers who may be interested in adjustable windows, this paper will draw a comparison on spectral characteristics among adjustable windows. The main work is organized as three parts: (1)The Section Two is to describe adjustable windows with analytic expressions suitable for any window length; (2)The Section Three is to compute and to fit the spectral characteristics of adjustable windows through numerical experiments; (3)The Section Four is to compare the spectral characteristics of adjustable windows.

\section{General description of adjustable windows}

The Kaiser and Cosh window. An analytic expression of the Kaiser window[13] for any window length is shown as:

$$
w_{\mathrm{k}}(n)=\left\{\begin{array}{l}
I_{0}\left[\alpha_{\mathrm{k}} \sqrt{1-\left(\frac{n-M}{M}\right)^{2}}\right]\left(I_{0}\left[\alpha_{\mathrm{k}}\right]\right)^{-1}, \quad 0 \leq n \leq N-1 \\
0, \quad \text { otherelse }
\end{array}\right.
$$

where $N$ is the window length, $M=(N-1) / 2$, and $k$ is the adjustable parameter, and $I_{0}[x]$ is the zeroth-order modified Bessel function of the first kind which can be described by the power series expansion as 


$$
I_{0}(x)=1+\sum_{k=1}^{\infty}\left[\frac{1}{k !}\left(\frac{x}{2}\right)^{k}\right]^{2}
$$

Generally, the total power series expansion was approximated to the sum of part power series for finite computation cost. From Eq.(1), an analytic expression of the Cosh window for any window length is suggested as:

$$
w_{c}(n)=\left\{\begin{array}{l}
\cosh \left[\alpha_{c} \sqrt{1-\left(\frac{n-M}{M}\right)^{2}}\right]\left(\cosh \left[\alpha_{c}\right]\right)^{-1}, \quad 0 \leq n \leq N-1 \\
0, \quad \text { otherelse }
\end{array}\right.
$$

where $\alpha_{c}$ is the adjustable parameter, and $\cosh (\mathrm{x})$ is the cosine hyperbolic function.

The ultraspherical, Dolph-Chebyshev, Saramäki window. An analytic expression of the right-sided ultraspherical window [17] for any window length is shown as:

$$
w(n)=\frac{A}{p-n} C_{p-n-1}^{\mu+p-n-1} \cdot \sum_{m=0}^{n} C_{n-m}^{\mu+n-1} C_{m}^{p-n} T^{m}
$$

where $0 \leq n \leq N-1$, and

$$
\begin{aligned}
& A= \begin{cases}\mu x_{\mu}^{p}, & \text { if } \mu>-1, \mu \neq 0 \\
x_{\mu}^{p}, & \mu=0\end{cases} \\
& T=1-x_{\mu}^{-2} \\
& P=N-1
\end{aligned}
$$

where the binomial coefficients can be calculated

$$
C_{0}^{m}=1, \quad C_{n}^{m}=\frac{m(m-1) \mathrm{L}(m-n+1)}{n !} \text { if } n \geq 1
$$

The independent parameter $\chi_{\mu}$ can be calculated:

$$
x_{\mu}=\frac{x_{N-1,1}^{(\mu)}}{\cos (\beta \pi / N)}
$$

where , $\beta \geq 1, \beta \neq(0.5+k) \cdot N, k \in \mathbf{Z}^{+}$, and $x_{N-1,1}^{(\mu)}$, which is estimated through some iterative algorithm[16], is the largest zero point of the ultraspherical polynomial $C_{N-1,1}^{(\mu)}$. Then there are three independent parameters $N, \beta$ and $\mu$ that determine the ultraspherical window. Surprisingly, the Dolph-Chebyshev window and Saramäki window are special cases of the ultraspherical windows with $\mu=0$ and $\mu=1$ respectively[16]. Moveover, the $x_{N-1,1}^{(0)}$ and $x_{N-1,1}^{(1)}$ can be denoted as

$$
\begin{aligned}
& x_{N-1,1}^{(0)}=\cos \left(\frac{\pi}{2(N-1)}\right) \\
& x_{N-1,1}^{(1)}=\cos (\pi / N)
\end{aligned}
$$

\section{Computation and fits of spectral characteristics}

The Kaiser window with length $N=11$ and adjustable parameter $\alpha_{\mathrm{k}}=1.1$ is taken as an example to illuminate some spectral characteristics parameters.

The spectral characteristics of window consist of main-lobe width, ripple ratio, side-lobe roll-off ratio, etc. The main-lobe width, which is denoted as $B=2 \omega_{0}$, is usually defined as the interval between angular frequency $-\omega_{0}$ and $+\omega_{0}$ where the main-lobe of amplitude spectrums decrease to zero[12]. The ripple ratio is defined as the ratio of the maximum amplitude of side-lobes( the first side-lobes in Fig. 1) to the amplitude of main-lobe, and it is denoted as $\mathrm{r}$, or in the form of $R=20 \lg (r)$. The side-lobe roll-off ratio is defined as ratio of the amplitude. of the furthest side-lobe to that of the first side-lobe, and it is denoted as s or in the form of $S=20 \lg (s)$. Besides, a new parameter is defined as the ratio of 
the main-lobe energy to whole bands energy in this paper for observing how close a window approximates to the discrete prolate functions,

$$
q=\frac{\int_{0}^{\omega_{0}}\left\|W\left(e^{j \omega}\right)\right\|^{2} \mathrm{~d} \omega}{\int_{0}^{\pi}\left\|W\left(e^{j \omega}\right)\right\|^{2} \mathrm{~d} \omega}
$$

Namely it is the ratio of energy concentrating in $|\omega| \leq \omega_{0}$ to total energy in $|\omega| \leq \pi$, or in the form of $Q=10 \lg (q)$, and $W\left(e^{j \omega}\right)$ is the Fourier transform of window coefficients. Obviously, the larger ratio $q$ is given, the closer approximation to the discrete prolate functions will be achieved. The coefficients and their Fourier transform are computed through numerical experiments, and the frequency interval is set $0.0001 \pi$ for calculating $W\left(e^{j \omega}\right)$ and fitting the spectral characteristics. For example, the spectral characteristics of the Kaiser window shown in Fig. 1 can be obtained $B=1.2246 \mathrm{rad} / \mathrm{s}, R=-15.0915 \mathrm{~dB}$, $S=-7.8122 \mathrm{~dB}, Q=-0.2526 \mathrm{~dB}$.

Subscripts 'k', 'c','d','s' of spectral characteristics stand for the Kaiser, Cosh, Dolph-Chebyshev, Saramaki and ultraspherical window $(\mu=2)$ respectively, such as the main-lobe width $B_{\mathrm{k}}, B_{\mathrm{c}}, B_{\mathrm{d}}, B_{\mathrm{s}}$ and, $B_{\mathrm{u} 2}$.

\section{Kaiser and Cosh window.}

Kaiser window. The coefficients of all Kaiser windows with parameters $15 \leq N \leq 512$ and $0.1 \leq \alpha_{\mathrm{k}} \leq 15$ were computed with MATLAB 7.8.0 according to Eq.(1-2), then the Fourier transforms and the spectral characteristics were calculated respectively. We set the interval of parameter $\alpha_{k}$ as 0.1 for computing coefficients of all the windows, and the total power series expansion was approximate to the sum of 70 orders power series. The black curve that the product of main-lobe width $B_{\mathrm{k}}$ and parameter $M$ versus the adjustable parameter $\alpha_{\mathrm{k}}$ for every window length $N$ is shown in Fig.2, and the black curve that the ripple ratio versus the adjustable parameter $\mathrm{k}$ is shown in Fig.3. There are 498 black curves both in Fig.2 and Fig.3.

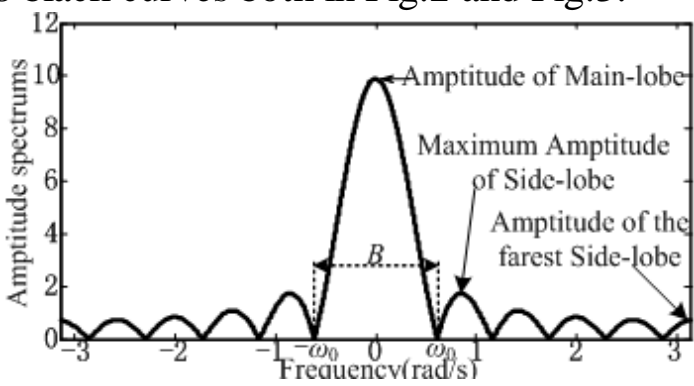

Fig. 1. Amplitude spectrums of a Kaiser window

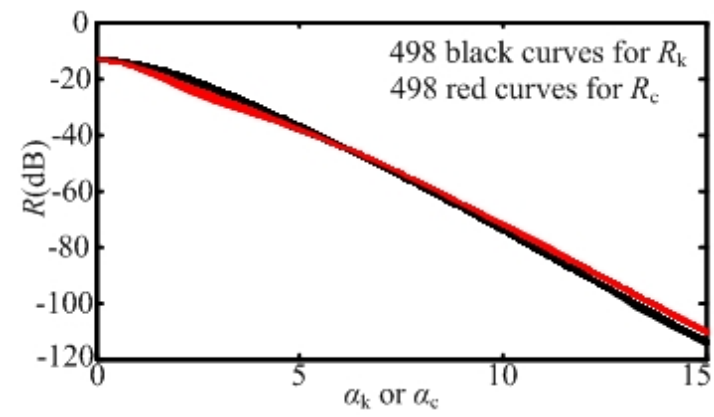

Fig. 3. $R$ of Kaiser and Cosh window vs $\alpha$ for every $N$

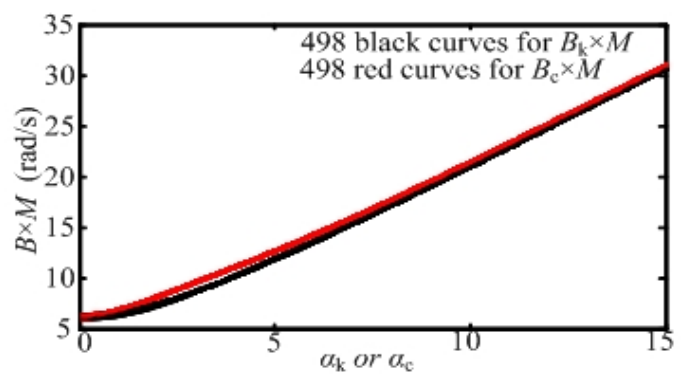

Fig. 2. $M B$ of Kaiser and Cosh window vs $\alpha$ for every $N$

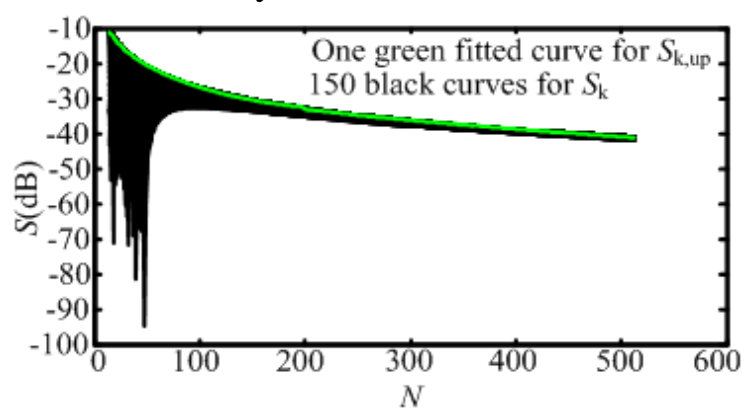

Fig. 4. $S_{\mathrm{k}}$ of Kaiser window vs $N$ for every $\alpha_{\mathrm{k}}$ 


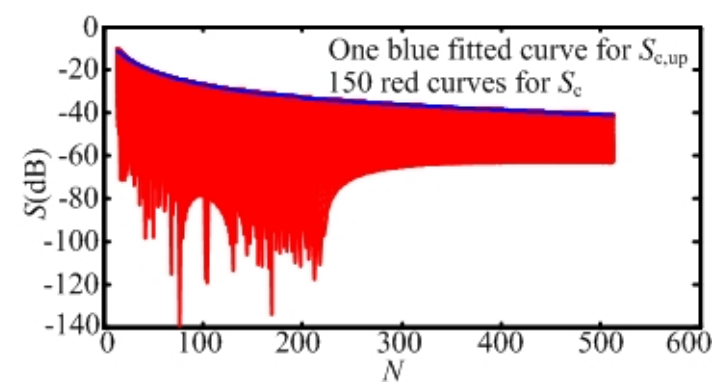

Fig. 5. $S_{\mathrm{c}}$ of Cosh window vs $N$ for every $\alpha_{c}$

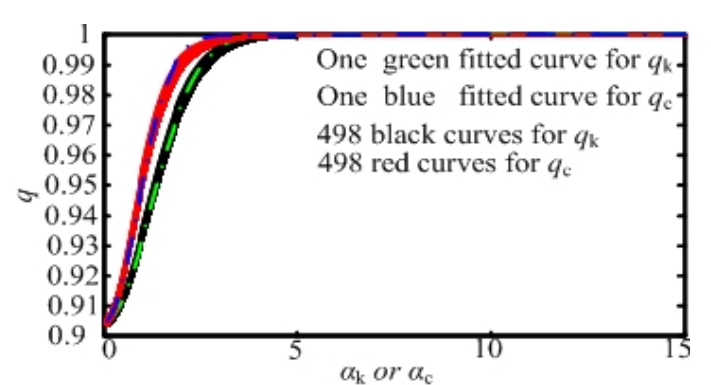

Fig. 6. $q$ of Kaiser and Cosh window vs $\alpha$ for every $N$

The closed form formulas of main-lobe width and ripple ratio hold for the analog Kaiser window can be expected to hold for the digital Kaiser window except for small $N$ or small $\alpha_{\mathrm{k}}$ [12],

$$
\begin{aligned}
& f_{0}=\frac{1}{2 \pi \tau} \sqrt{\pi^{2}+\alpha_{\mathrm{k}}^{2}} \\
& -R_{\mathrm{k}}=20 \lg \left|\frac{\sinh \left(\alpha_{\mathrm{k}}\right)}{0.217234 \alpha_{\mathrm{k}}}\right|
\end{aligned}
$$

Where $f_{0}=\omega_{0} / 2 \pi t_{s}$, and $t_{s}$ is the sample interval. The Eq. (10-11) can be converted into

$$
\begin{aligned}
& B_{\mathrm{k}}=\frac{2}{M} \sqrt{\pi^{2}+\alpha_{\mathrm{k}}^{2}} \\
& R_{\mathrm{k}}=20 \lg \left|\frac{0.217234 \alpha_{\mathrm{k}}}{\sinh \left(\alpha_{\mathrm{k}}\right)}\right|
\end{aligned}
$$

The relative deviation between the spectral characteristics computed with Eq.(12-13) and ones obtained by experiments decrease rapidly when $N<67$, and they are less than $\pm 9 \%$ for $N<67$ while less than $\pm 2 \%$ for $N \geq 67$. Furthermore, the experiment results changed little when the total power series expansion(2) was approximate to the sum of 80 or 90 orders power series. The curve that the side-lobe roll-off ratio versus $N$ for every $\alpha_{\mathrm{k}}$ is shown in Fig. 4 where a clear upper bound curve which was fitted by a green curve exists. The fitted expression is

$$
S_{\mathrm{k}, \text { up }}=80.5 N^{-0.1}-72+\Delta_{\mathrm{k}, \text { up }}
$$

where

$$
\Delta_{\text {k,up }}=\left\{\begin{array}{l}
0.0002 N^{2}-0.0814 N+0.6540, \quad N \leq 200 \\
-0.0122 N-6.0257, \quad N>200
\end{array}\right.
$$

The relative deviation between the upper bound of side-lobe roll-off ratio and the fitted one is less than $\pm 1 \%$ for $N \geq 25$. The black curve of main-lobe energy ratio versus the adjustable parameter $\alpha_{k}$ for every $N$ is shown in Fig6, and it can be fitted with a green curve which is described of

$$
q_{\mathrm{k}}=10^{-0.0436 \cdot e^{-\alpha_{k}^{2} / 2.8}}
$$

For any window length $N$ and adjustable parameter $\alpha_{\mathrm{k}}$, the relative deviation between $q_{\mathrm{k}}$ and the fitted one is less than $0.4869 \%$.

Cosh window. Similarly, we computed the coefficients of all Cosh windows with parameters $15 \leq N \leq 512$ and $0.1 \leq \alpha_{c} \leq 15$ according to Eq.(3), and the interval of $\alpha_{c}$ was also set as 0.1 . The red curve that the product of main-lobe width $B_{\mathrm{c}}$ and parameter $M$ versus the adjustable parameter $\alpha_{\mathrm{c}}$ for every window length $N$ is also shown in Fig.2, and the red curve that the ripple ratio $R_{\mathrm{c}}$ versus the adjustable parameter $\alpha_{c}$ is also shown in Fig.3. The red curve that the side-lobe roll-off ratio $S_{\mathrm{c}}$ versus $N$ for every $\alpha_{c}$ is shown in Fig.5.

The piecewise of main-lobe width curve can be fitted with 


$$
B_{c}= \begin{cases}\left(0.6507 \alpha_{c}+6.1132\right) / M, & 0<\alpha_{c} \leq 1 \\ \left(1.3396 \alpha_{c}+5.4388\right) / M, & 1<\alpha_{c} \leq 2 \\ \left(1.7764 \alpha_{c}+3.8584\right) / M, & 2<\alpha_{c} \leq 15\end{cases}
$$

For any window length $N$ and adjustable parameter $\alpha_{c}$, therelative deviation between $B_{\mathrm{c}}$ and the fitted one is less than $6.7763 \%$.

The piecewise of ripple ratio curve can be fitted with

$$
R_{\mathrm{c}}= \begin{cases}-2.2269 \alpha_{\mathrm{c}}-12.9326, & 0<\alpha_{\mathrm{c}} \leq 0.6 \\ -5.4986 \alpha_{\mathrm{c}}-10.3859, & 0.6<\alpha_{\mathrm{c}} \leq 5 \\ -7.3660 \alpha_{\mathrm{c}}-1.00000, & 5<\alpha_{\mathrm{c}} \leq 15\end{cases}
$$

For any window length $N$ and adjustable parameter $\alpha_{\mathrm{c}}$, the relative deviation between $R_{\mathrm{c}}$ and the fitted one is less than $6.8637 \%$.

It is interesting that the upper bound of $S_{\mathrm{c}}$ is almost the same as the one of $S_{\mathrm{k}}$, so the upper bound $S_{\mathrm{c} \text {,up }}$ can be also fitted with the upper bound $S_{\mathrm{k} \text {, up }}$, which is shown as a blue curve.

And, the main-lobe energy ratio can be fitted with

$$
q_{\mathrm{c}}=10^{-0.0436 \cdot e^{-\alpha_{\mathrm{c}}^{2} / 1.46}}
$$

For any window length $N$ and adjustable parameter $\alpha_{\mathrm{c}}$, the relative deviation between $q_{\mathrm{c}}$ and the fitted one is less than $0.5473 \%$, and the blue fitted curve is plotted in Fig.6.

\section{Ultraspherical window.}

For a fixed $N$ and a prescribed side-lobe roll-off ratio $S$, one can select the parameter $\mu$ appropriately. To generate an ultraspherical window with $\mu$ and $N$ fixed and a prescribed main-lobe half width of $\omega_{0}$, one can select the parameter $x_{\mu}$ or appropriately. To generate an ultraspherical window with $\mu$ and $N$ fixed and a prescribed ripple ratio $R$, one can select the parameter $x_{\mu}$ appropriately[16]. In other words, the side-lobe roll-off ratio $S$ may be determined by the window length $N$ and parameter $\mu$, and both the main-lobe width $B$ and ripple ratio $R$ may be determined by the window length $N$, parameter $\mu$ and $\beta$.

As the side-lobe roll-off ratio $S$ relates to $\mu$ and $N$ except $\beta$, we computed the coefficients of all ultraspherical windows with parameters $15 \leq N \leq 512$ and $-1<\mu \leq 10$ according to Eq.(4) in the condition of $\beta=1$. The bound of $\mu$ is suggested by S.W.A. Bergen[16] and the interval of $\mu$ was set as 0.01 . The curves of the side-lobe roll-off ratio versus the adjustable parameter $\mu$ for $N=16, N=32$ and $N=48$ are shown in Fig.7.

In fact, the curve of the side-lobe roll-off ratio versus the adjustable parameter $\mu$ for every $N$ can be fitted with function

$$
s=a(N) e^{b(N) \mu}+c(N) e^{d(N) \mu}
$$

where $a(N), b(N), c(N)$ and $d(N)$ are functions of window length $N$. Their values for $N=16 ; 32 ; 64$; $128 ; 256 ; 512$ are enumerated in Table 1

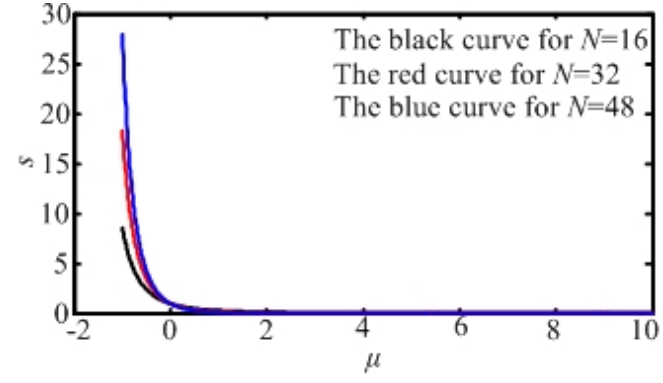

Fig. 7. $s$ of ultraspherical window vs $\mu$ for some $N$

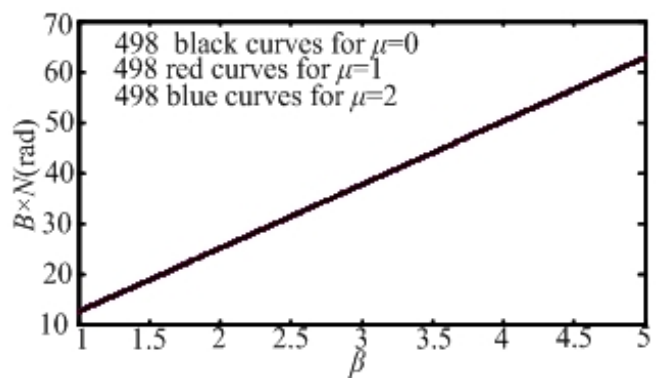

Fig. 8. $B \cdot N$ of ultraspherical window vs $\beta$ for some $N$ 


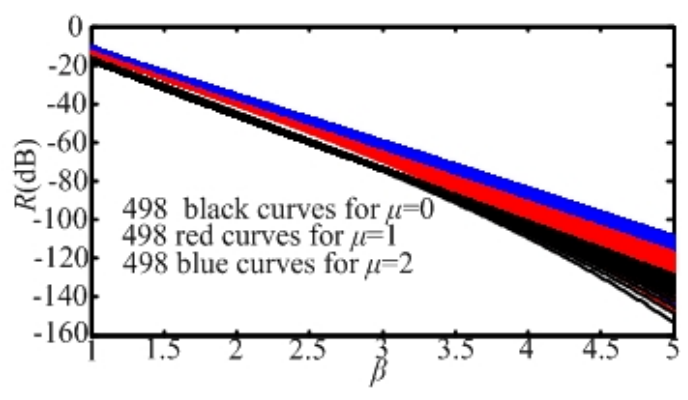

Fig. 9. $R$ of ultraspherical window vs $\beta$ for some $N$

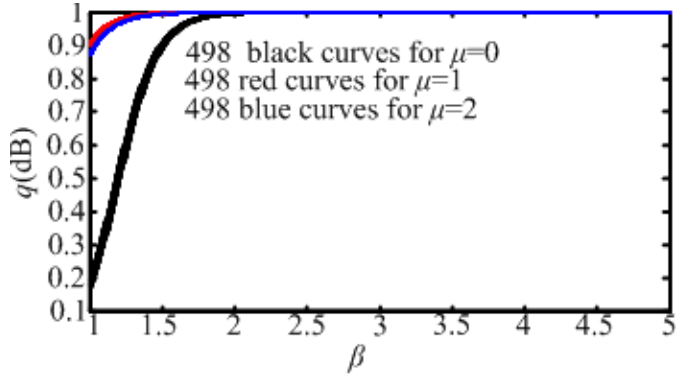

Fig. 10. $q$ of ultraspherical window vs $\beta$ for some $N$

Table.1 Values of $a(N), b(N), c(N)$ and $d(N)$ for some window length

\begin{tabular}{c|c|c|c|c|c|c}
\hline$N$ & 16 & 32 & 64 & 128 & 256 & 512 \\
\hline$a(N)$ & 0.1557 & 0.06705 & 0.03956 & 0.02701 & 0.02009 & 0.01188 \\
$b(N)$ & -3.709 & -5.166 & -6.311 & -7.316 & -8.244 & -9.359 \\
$c(N)$ & 0.8409 & 0.954 & 0.9693 & 0.9628 & 0.9509 & 0.9319 \\
$d(N)$ & -1.032 & -2.021 & -2.896 & -3.712 & -4.493 & -5.305 \\
\hline
\end{tabular}

The relative deviation between $s$ and the one fitted is less than5.6076\% for $\mu \leq 0$, and the absolute deviation between $\mathrm{s}$ and the one fitted is less than 0.0561 for $\mu>0$.

Both the main-lobe width $B$ and ripple ratio $R$ are relate to the window length $N$, parameter $\mu$ and $\beta$ simultaneously. As a result, the computation cost of $B$ and $R$ with different $N, \mu$ and $\beta$ is huge, and it is difficult to fit the experiment data of $B$ and $R$ with functions of three variables $N, \mu$ and $\beta$. So we only try to obtain the main-lobe width $B$ and ripple ratio $R$ of ultraspherical window at some special cases, such as $\mu=0, \mu=1$ and $\mu=2$. As we know, the Dolph-Chebyshev and Saramäki window are special cases of ultraspherical window with parameter $\mu=0$ and $\mu=1$ respectively.

Dolph-Chebyshev window. The coefficients of all ultraspherical windows with parameters $\mu=0$, $15 \leq N \leq 512$ and $1 \leq \beta \leq 5$ were computed according to Eq.(4), and the interval of $\beta$ was set as 0.01 . The black curve of the product of main-lobe width $B_{\mathrm{d}}$ and parameter $N$ versus the adjustable parameter for every window length $N$ is shown in Fig.8, and the black curve that the ripple ratio $R_{\mathrm{d}}$ versus the adjustable parameter $\beta$ is shown in Fig.9.

The curves of $B_{\mathrm{d}}$ versus the adjustable parameter $\beta$ for every window length $N$ can be fitted with $B_{\mathrm{d}}=(12.5678 \beta-0.0001) / N$

For any $N$ and $\beta$, the relative deviation between $B_{\mathrm{d}}$ and the fitted one is less than $1.2251 \%$.

The close form expressions for the ripple ratio $R_{\mathrm{d}}$ of Dolph- Chebyshev window is given as[8]:

$r=1 / T_{N-1}\left(x_{0}\right)$

where[16]

$x_{0}=x_{\mu}=\frac{x_{N-1,1}^{(0)}}{\cos (\beta \pi / N)}$

The expressions equal the following expressions:

$R_{\mathrm{d}}=20 \lg \left[1 / T_{N-1}\left(x_{0}\right)\right]$

The relative deviation between $\mathrm{Rd}$ and the fitted one is less than $2.3602 \times 10^{-5} \%$. However, the expression is complex though it is accurate enough. We can approximate it with a linear function for large window length $N$. For example,

$R_{\mathrm{d}}=\left\{\begin{array}{l}-29.6914 \beta+12.1228, \quad 1 \leq \beta<1.5 \\ -27.6316 \beta+8.7251, \quad 1.5 \leq \beta<5\end{array}\right.$

For any window length $N \geq 32$ and $\beta$, the relative deviation between $R_{\mathrm{d}}$ and the fitted one is less than $5.1741 \%$.

The curves of $q_{\mathrm{d}}$ versus the adjustable parameter $\beta$ for every window length $N$ can be fitted with 


$$
q_{\mathrm{d}}=10^{-\left(190 N^{-1}+1+\Delta\right)^{-1} \beta^{-8}}
$$

where

$$
\Delta= \begin{cases}0.1167 N-2.8167, & N \leq 25 \\ 0.0139 N-0.2030, & 25<N \leq 45 \\ -0.0023 N+0.4301, & N>45\end{cases}
$$

For small $N$, the relative deviation between $q_{\mathrm{d}}$ and the fitted one is small, for example, less than $6.7486 \%$ for $N \leq 135$. However, the relative deviation become large for large $N$ and little $\beta$, for example, larger than $9.6959 \%$ and less than $85.1732 \%$ for $N>135$ and $\beta \leq 1.3$.

Saramäki window. Experiments were performed on the Saramäki window in the same way as the Dolph-Chebyshev window, and others parameters were fixed except that the parameter $\mu$ was set $\mu=$ 1. The red curve that the product of main-lobe width $B_{\mathrm{s}}$ and parameter $N$ versus the for every $N$ is shown in Fig.8, and the red curve that the ripple ratio $R_{\mathrm{S}}$ versus is shown in Fig.9. The fitted expressions for the main-lobe width of Saramäki window are given as:

$$
B_{\mathrm{s}}=(12.5674 \beta-0.0001) / \mathrm{N}
$$

For any $N$ and , the relative deviation between $B_{\mathrm{s}}$ and the fitted one is less than $1.2223 \%$. Similarly, the ripple ratio $R_{\mathrm{s}}$ can be approximated with a linear function for large $N$,

$$
R_{\mathrm{s}}= \begin{cases}-27.1126 \beta+13.7514, & 1 \leq \beta<1.5 \\ -25.8526 \beta+11.9771, & 1.5 \leq \beta<5\end{cases}
$$

For any $N \geq 32$ and $\beta$, the relative deviation between $R_{\mathrm{s}}$ and the fitted one is less than $3.9771 \%$. The curves of $q_{\mathrm{d}}$ versus for every $N$ can be fitted with

$$
q_{\mathrm{s}}=10^{-1 /\left(22.6 \beta^{8}\right)}
$$

For any $N$ and $\beta$, the relative deviation between $q_{\mathrm{s}}$ and the fitted one is less than $0.2337 \%$.

Ultraspherical window $(\boldsymbol{\mu}=\mathbf{2})$. Experiments were performed on the ultraspherical window $(\mu=2)$ in the same way, and others parameters were fixed except setting $\mu=2$. The blue curve that the product of main-lobe width $B_{\mathrm{u} 2}$ and parameter $N$ versus for every $N$ is shown in Fig.8, and the blue curve that the ripple ratio $R_{\mathrm{u} 2}$ versus is shown in Fig.9. The fitted expressions for $B_{\mathrm{u} 2}$ is given as:

$$
B_{\mathrm{u} 2}=(12.5673 \beta-0.0001) / N
$$

For any $N$ and $\beta$, the relative deviation between $B_{\mathrm{u} 2}$ and the fitted one is less than $1.2208 \%$, and the ripple ratio $R_{\mathrm{u} 2}$ can be approximated with a linear function for large $N$.

$$
R_{\mathrm{u} 2}= \begin{cases}-25.3840 \beta+14.7831, & 1 \leq \beta<1.5 \\ -24.4176 \beta+13.6835, & 1.5 \leq \beta<5\end{cases}
$$

For any $N \geq 32$ and , the relative deviation between $R_{\mathrm{u} 2}$ and the fitted one is less than $6.5430 \%$. The main-lobe energy ratio $q_{\mathrm{u} 2}$ can be approximated with

$$
q_{\mathrm{u} 2}=10^{-1 /\left(16.5 \beta^{8}\right)}
$$

For any $N$ and $\beta$, the relative deviation between $q_{\mathrm{u} 2}$ and the fitted one is less than $3.4360 \%$, and it becomes less than $1 \%$ for $\beta \geq 1.16$.

\section{Comparisons of spectral characteristics}

In to spectrum analysis, the main-lobe width determines the ability to resolve adjacent spectral lines, and the ripple ratio determines the leakage or interaction between spectral lines[12]. For beamforming applications, higher side-lobe roll-off ratio means better performance of rejecting far end interferences[6]. In the application of FIR filter design, the main-lobe width mainly determines the transition band of filter, and the ripple ratio and side-lobe roll-off ratio commonly determine the ripple and stopband attenuation. Windows with narrow main-lobe, low ripple-ratio and low side-lobe roll-off 
ratio are expected generally in applications. The performance of spectral characteristics for kinds of adjustable windows were shown by comparisons.

main-lobe width versus ripple ratio. The experiment results show that the main-lobe width decreases when the ripple ratio or window length increases for all adjustable windows. The fitted expressions of these curves that the main-lobe width versus the ripple ratio are given as follows. For Kaiser window,

$$
B_{\mathrm{k}}= \begin{cases}\left(-0.2434 R_{\mathrm{k}}+2.9203\right) / M, & R_{\mathrm{k}} \leq-40 \mathrm{~dB} \\ \left(-0.4774 R_{\mathrm{k}}+6.1352\right) / M, & R_{\mathrm{k}}>-40 \mathrm{~dB}\end{cases}
$$

For any $N$ and $R_{\mathrm{k}}$, the relative deviation between $B_{\mathrm{k}}$ and the fitted one is less than $5.3448 \%$. For Cosh windows

$$
B_{\mathrm{c}}= \begin{cases}\left(-0.2510 R_{\mathrm{c}}+3.2979\right) / M, & R_{\mathrm{c}} \leq-40 \mathrm{~dB} \\ \left(-0.5296 R_{\mathrm{c}}+5.2544\right) / M, & R_{\mathrm{c}}>-40 \mathrm{~dB}\end{cases}
$$

For any $N$ and $R_{\mathrm{c}}$, the relative deviation between $B_{\mathrm{c}}$ and the fitted one is less than $6.8170 \%$. For Dolph-Chebyshev windows,

$$
B_{\mathrm{d}}=(12.5678 \beta-0.0001) / N
$$

where

$$
\beta=\frac{N}{\pi} \cos ^{-1} \frac{\cos [\pi / 2(N-1)]}{\cosh \left(\frac{\cosh ^{-1}\left(1 / r_{\mathrm{d}}\right)}{N-1}\right)}
$$

For any $N$ and $r_{\mathrm{d}}$, the relative deviation between $B_{\mathrm{d}}$ and the fitted one is less than $1.2553 \%$, but the expression is complex. For large $N$, a simpler linear function can be adopted to fit $B_{\mathrm{d}}$

$$
B_{\mathrm{d}}= \begin{cases}\left(3.9684-0.4548 R_{\mathrm{d}}\right) / N, & R_{\mathrm{d}} \leq-32.4143 \mathrm{~dB} \\ \left(5.1312-0.4233 R_{\mathrm{d}}\right) / N, & R_{\mathrm{d}}>-32.4143 \mathrm{~dB}\end{cases}
$$

For any $N \geq 32$ and $R_{\mathrm{d}}$, the relative deviation between $B_{\mathrm{d}}$ and the fitted one is less than $3.2001 \%$. For Saramäki windows with $N \geq 32$,

$$
B_{\mathrm{s}}= \begin{cases}\left(5.9437-0.4840 R_{\mathrm{s}}\right) / N, & R_{\mathrm{s}} \leq-60 \mathrm{~dB} \\ \left(6.0200-0.4840 R_{\mathrm{s}}\right) / N, & R_{\mathrm{s}}>-60 \mathrm{~dB}\end{cases}
$$

For any $N \geq 32$ and $R_{\mathrm{s}}$, the relative deviation between $B_{\mathrm{s}}$ and the fitted one is less than $3.6752 \%$. For the ultraspherial windows $(\mu=2)$ with $N \geq 32$,

$$
B_{\mathrm{u} 2}= \begin{cases}\left(6.9095-0.5175 R_{\mathrm{u} 2}\right) / N, & R_{\mathrm{u} 2} \leq-60 \mathrm{~dB} \\ \left(7.5109-0.5093 R_{\mathrm{u} 2}\right) / N, & R_{\mathrm{u} 2}>-60 \mathrm{~dB}\end{cases}
$$

For any $N \geq 32$ and $R_{\mathrm{u} 2}$, the relative deviation between $B_{\mathrm{u} 2}$ and the fitted one is less than $5.9722 \%$. It is found that $B_{\mathrm{d}}<B_{\mathrm{s}}<B_{\mathrm{k}}<B_{\mathrm{c}}<B_{\mathrm{u} 2}$ if given a fixed window length and a fixed ripple ratio. The curves of mainlobe width versus ripple ratio of adjustable windows for a fixed window length $\mathrm{N}=63$ is shown in Fig.11,

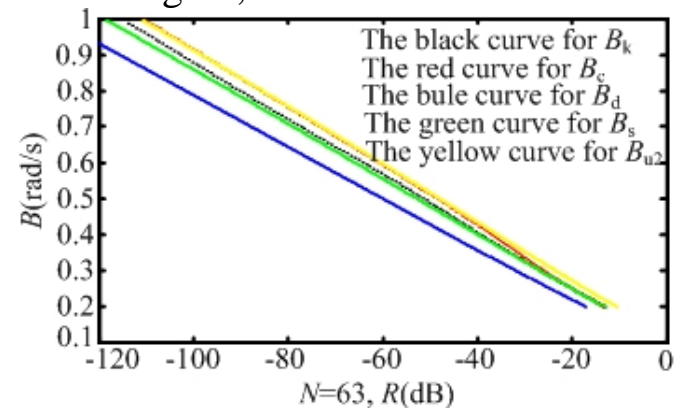

Fig. 11. $B$ vs $R$ of adjustable windows for the fixed $N=63$

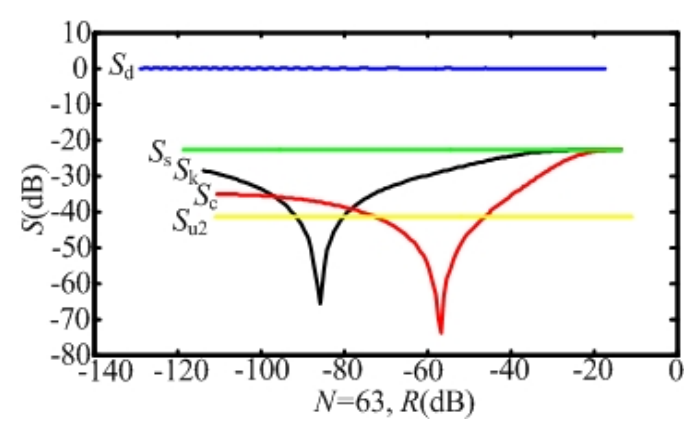

Fig. 12. $S$ vs $R$ of adjustable windows for the fixed $N=63$ 


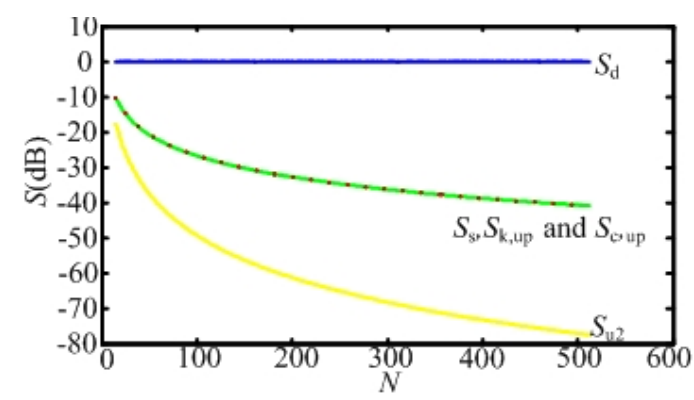

Fig. 13. $S$ vs $N$ of adjustable windows

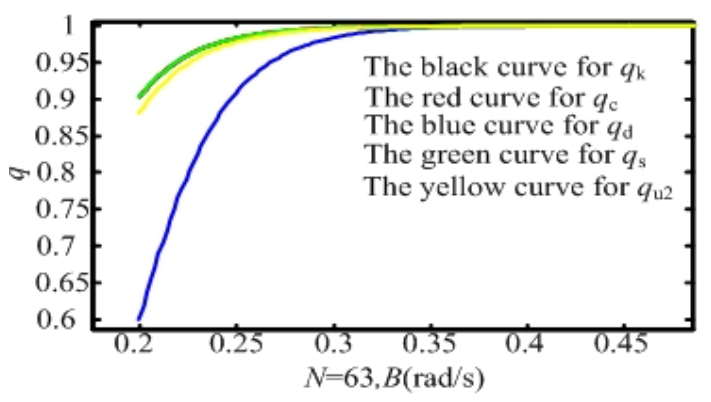

Fig. 14. $q$ vs $B$ of adjustable windows for the fixed $N=63$

Note that the $B_{\mathrm{k}}$ becomes closer to $B_{\mathrm{s}}$ as the window length increases. For example, the relative difference between $B_{\mathrm{k}}$ and $B_{\mathrm{s}}$ is less than $0.0075 \%$ for the fixed window length $\mathrm{N}=512$ and any fixed ripple ratio.

side-lobe roll-off ratio versus ripple ratio. The curves of $S_{\mathrm{k}}$ versus $R_{\mathrm{c}}$ and $S_{\mathrm{c}}$ versus $R_{\mathrm{c}}$ are not regular for some fixed $N$, and the ones of $S_{\mathrm{d}}$ versus $R_{\mathrm{d}}, S_{\mathrm{s}}$ versus $R_{\mathrm{s}}$ and $S_{\mathrm{u} 2}$ versus $R_{\mathrm{u} 2}$ are horizontal lines for any fixed $N$. Windows with $N=63$ are taken as examples in Fig.12

However, the upper bound of $S_{\mathrm{k}}$ and $S_{\mathrm{c}}$ are certain for every $N$, and it is found that the $S_{\mathrm{d}}, S_{\mathrm{s}}$ and $S_{\mathrm{u} 2}$ are independent with ripple ratio for fixed window length. The $S_{\mathrm{d}}, S_{\mathrm{s}}, S_{\mathrm{u} 2}$ as well as the upper bound of $S_{\mathrm{k}}$ and $S_{\mathrm{c}}$ for every $N$ are shown in Figure.13.

As shown in Fig13, the curve of $S_{\mathrm{s}}$ versus $N$ are almost the same as the ones of the upper bound of $S_{\mathrm{k}}$ and $S_{\mathrm{c}}$, and the relative deviations among them is less than $0.001 \%$. Besides, we can find there is $S_{\mathrm{u} 2}$ $<S_{\mathrm{s}}<S_{\mathrm{d}}$ for any fixed $N$.

main-lobe energy ratio versus main-lobe width. The main-lobe energy ratio has positive correlation with the main-lobe width, and the former gets to one as the latter increases. An example is shown in Fig.14. For any fixed window length and main-lobe width, the main-lobe energy ratio can be fitted as the following. For Kaiser windows,

$$
q_{\mathrm{k}}=10^{-0.0436 \cdot e^{-\left(\pi^{2}-0.25 \mathrm{M}^{2} B_{\mathrm{k}}^{2}\right) / 2.8}}
$$

The relative deviation between $q_{\mathrm{k}}$ and the fitted one is less than $5.5937 \%$ for any $M$ (or $N$ ) and $B_{\mathrm{k}}$, and it becomes less than $1 \%$ for $B_{\mathrm{k}}>1 \mathrm{rad} / \mathrm{s}$. For Cosh windows,

$$
q_{\mathrm{k}}=10^{-0.0436 X}
$$

where

$$
X=\left\{\begin{array}{lc}
e^{-1.6177\left(M B_{\mathrm{c}}-6.1132\right)^{2}}, & 6.1132<M B_{\mathrm{c}} \leq 6.7639 \\
e^{-0.3817\left(M B_{\mathrm{c}}-5.4388\right)^{2}}, & 6.7639<M B_{\mathrm{c}} \leq 8.1180 \\
e^{-2.171\left(M B_{\mathrm{c}}-3.8584\right)^{2}}, & 8.1180<M B_{\mathrm{c}} \leq 30.5044
\end{array}\right.
$$

The relative deviation between $q_{\mathrm{c}}$ and the fitted one is less than $3.5956 \%$ for any parameter $M$ (or $N$ ) and $B_{\mathrm{c}}$, and it becomes less than $1 \%$ for $B_{\mathrm{c}}>1 \mathrm{rad} / \mathrm{s}$. For Dolph-Chebyshev windows,

$q_{\mathrm{d}}=10^{-12.5678^{8}\left(N B_{\mathrm{d}}+0.0001\right)^{-8}\left(190 N^{-1}+1+\Delta\right)^{-1}}$

where

$$
\Delta= \begin{cases}0.1167 N-2.8167, & N \leq 25 \\ 0.0139 N-0.2030, & 25<N \leq 45 \\ -0.0023 N+0.4301, & N>45\end{cases}
$$

The relative deviation between $q_{\mathrm{d}}$ and the fitted one is less than $7.3189 \%$ for $N \leq 135$, but it becomes larger for bigger $N$ and less $B_{\mathrm{d}}$, such as more than $8.0749 \%$ for $N>315$ and $B_{\mathrm{d}}<0.0415 \mathrm{rad} / \mathrm{s}$. For Saramäki windows,

$$
q_{\mathrm{s}}=10^{-12.5674^{8}\left(N B_{\mathrm{s}}+0.0001\right)^{-8} / 22.6}
$$


The relative deviation between $q_{\mathrm{s}}$ and the fitted one is less than $1.2566 \%$ for any parameter $\mathrm{N}$ and Bs. For ultraspherial windows $(\mu=2)$.

$$
q_{\mathrm{u} 2}=10^{-12.5673^{8}\left(N B_{\mathrm{u} 2}+0.0001\right)^{-8} / 16.5}
$$

The relative deviation between $q_{\mathrm{u} 2}$ and the fitted one is less than $5.2706 \%$ for any $N$ and $B_{\mathrm{u} 2}$,

For any fixed $N$ and main-lobe width, it is found that $q_{\mathrm{s}}$ and $q_{\mathrm{c}}$ are almost the same as the $q_{\mathrm{k}}$, which means the Saramäki and Cosh are like to the Kaiser windows that they both closely approximate to the prolate spheroidal wave function. Besides, there is $q_{\mathrm{d}}<q_{\mathrm{u} 2}<q_{\mathrm{s}}$, and the Dolph-Chebyshev windows are obviously not close to the prolate spheroidal wave function. Windows with $N=63$ are taken as examples in Fig.14, where, the black curve, red curve and green curve almost overlap, and the relative deviations among them is less than $0.01 \%$.

\section{Conclusion}

Analytic expressions which are suitable for any window length were adopted to compute the coefficients of adjustable windows including the Kaiser, Cosh, Dolph-Chebyshev, Saramäki and other ultraspherical windows. Well fits of the curves that the main-lobe width, ripple ratio, side-lobe roll-off ratio and main-lobe energy ratio versus corresponding adjustable parameters were performed. Furthermore, the curves that main-lobe width versus ripple ratio, side-lobe roll-off ratio versus ripple ratio and main-lobe energy ratio versus main-lobe width were fitted. The relative deviations between these spectral characteristics and the fitted ones are normally less than $\$ 7 \backslash \%$. In conclusion, some result were obtained through comparison:

1) The main-lobe widths of adjustable windows satisfy $B_{\mathrm{d}}<B_{\mathrm{s}}<B_{\mathrm{k}}<B \mathrm{c}<B_{\mathrm{u} 2}$ in condition with same window length and ripple ratio.

2) The side-lobe roll-off ratios of adjustable windows satisfy $S_{\mathrm{u} 2}<S_{\mathrm{s}}<S_{\mathrm{d}}=0 \mathrm{~dB}$ and $S_{\mathrm{s}} \approx S_{\text {c,up }} \approx S_{\mathrm{k} \text {,up }}$ in condition with same window length and ripple ratio, and the relative deviations among $S_{\mathrm{s}}, S_{\mathrm{c} \text {,up }}$, and $S_{\mathrm{k} \text {,up }}$ is less than $0.001 \%$ which means $S_{\mathrm{k}}, S_{\mathrm{c}}<S_{\mathrm{s}}<0 \mathrm{~dB}$. But the order of $S_{\mathrm{c}}, S_{\mathrm{k}}$ and $S_{\mathrm{u} 2}$ is uncertain.

3) The main-lobe energy ratio of adjustable windows satisfy $0<q_{\mathrm{d}}<q_{\mathrm{u} 2}<q_{\mathrm{s}}<1$ and $q_{\mathrm{s}} \approx q_{\mathrm{c}} \approx q_{\mathrm{k}}$ in condition with same window length and main-lobe width. The relative deviations among $q_{\mathrm{s}}, q_{\mathrm{c}}$, and $q_{\mathrm{k}}$ is less than $0.01 \%$ which means the Saramaki and Cosh window are both close to the prolate spheroidal wave function like the Kaiser window.

From above, it is found that the Saramaki window has better overall performance than other adjustable windows through comparing those spectral characteristics. The work in this paper may be helpful for some researchers who need choosing adjustable windows in signal spectral analysis, digital filter design and other occasions.

\section{References}

[1] Saramäki T. Finite impulse response filter design. In: Mitra SK and Kaiser JF, editors. In Handbook for Digital Signal Processing, New York: Wiley;1993.

[2] Dolph CL. A current distribution for broadside arrays which optimizes the relationship between beamwidth and side-lobe level. Proc IRE 1946, pp 335-348.

[3] Kaiser JF. Digital Filters. System Analysis by Digital Computer. Kuo FF and Kaiser JF, Eds. New York: Wiley;1966, pp. 218-285.

[4] Saramäki T. A class of window functions with nearly minimum sidelobe energy for designing FIR filters. In: Proceedings of the IEEE international symposium circuits and systems, Portland, Ore, USA, 359,362 (1989).

[5] Deczky AG. Unispherical windows. In: Proceedings of the IEEE international symposium circuits and systems, Sydney, NSW, Australia, 85,88 (2001). 
[6] Avcia K, Nacaroglub A. Cosh window family and its application to FIR filter design. Int. J. Electron. Commun. (AEU ) 63 (2009) 907-16

[7] Kraus,J.D. Antennas. 2nd ed. New York: McGraw-Hill; 1988, pp.892

[8] Lynch P. The Dolph-Chebyshev window: a simple optimal filter. Monthly Weather Review, 125 (1997) 655-60.

[9] Antoniou A. Digital Filters: Analysis, Design and Application. 2nd ed. New York: McGraw-Hill; 1993, pp.689.

[10] Slepian D, Pollak HO, Landau HJ. Prolate spheroidal wave functions, Fourier analysis, and uncertainty-I. Bell Syst. Tech. J. 40 (1961) 43-63.

[11] Kaiser JF. Nonrecursive digital filter design using I0-sinh window function. In: Proceedings of IEEE international symposium circuits and systems, SanFrancisco, CA, USA, 20,23(1974).

[12] Kaiser JF, Schafer RW. On the use of the I0-sinh window for spectrum analysis. IEEE Trans Acoustics Speech Signal Process. 28 (1980) 105-107.

[13] Oppenheim AV, Schafer RW. Discrete-time signal processing. 3nd ed. Upper Saddle River, N.J: Prentice Hall. 2010, pp.542-545.

[14] Abramowitz M, Stegun I. Handbook of Mathematical Functions. New York: Dover Publications; 1965.

[15] Bergen SWA, Antoniou A. Generation of ultraspherical window functions. In Proc. 11th European Signal Processing Conference (EUSIPCO 02), Toulouse, France, 607,610(2002).

[16] Bergen SWA, Antoniou A. Design of Ultraspherical Window Functions with Prescribed Spectral Characteristics. EURASIP Journal on Applied Signal Processing. 13 (2004) 2053-2065

[17] Bergen SWA, Antoniou A. Nonrecursive digital filter design using the ultraspherical window. In Proc. IEEE Pacific Rim Conference on Communications, Computers and Signal Processing (PACRIM 03), Victoria, BC, Canada, 260,263(2003).

[18] Harish K, Piyush K and Kaushal K, et al. Design And Performance of Finite impulse Response Filter Using Hyperbolic CosineWindow. ACEEE Int. J. on Communication. 2 (2011) 45-49.

[19] Prabhu K.M.M.Window Functions and Their Applications in Signal Processing. New York: CRC Press, 2014.

[20] Alia A.E and Amer R.Z. FIR Digital Filter Design By Using Windows Method With MATLAB. 14th international conference on Sciences and Techniques of Automatic control and computer engineering - STA’2013, Sousse, Tunisia, 282,287(2013).

[21] Hrishi R, Muhammad A.U. A Comparative Study on Window Functions for Designing Efficient FIR Filter. The 9th International Forum on Strategic Technology (IFOST),Coxs Bazar, Bangladesh, 91,96(2014). 